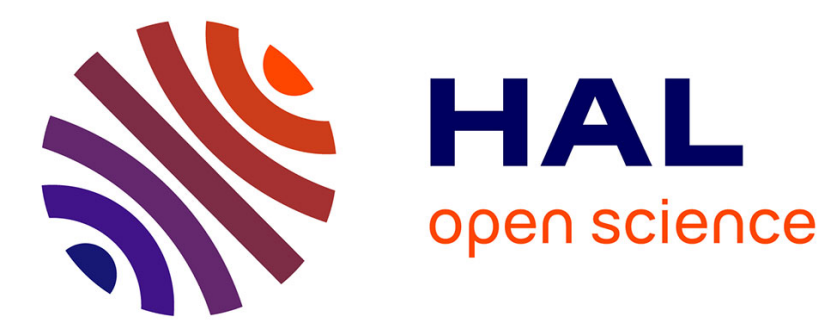

\title{
Médiateurs, pairs, interprètes au secours du soin
}

Nicolas Chambon, Véronique Traverso, Halima Zeroug-Vial

\section{To cite this version:}

Nicolas Chambon, Véronique Traverso, Halima Zeroug-Vial. Médiateurs, pairs, interprètes au secours du soin. Rhizome, 2020, Nº75-76 (1), pp.7. 10.3917/rhiz.075.0007 . halshs-03169205

\section{HAL Id: halshs-03169205 \\ https://shs.hal.science/halshs-03169205}

Submitted on 15 Mar 2021

HAL is a multi-disciplinary open access archive for the deposit and dissemination of scientific research documents, whether they are published or not. The documents may come from teaching and research institutions in France or abroad, or from public or private research centers.
L'archive ouverte pluridisciplinaire HAL, est destinée au dépôt et à la diffusion de documents scientifiques de niveau recherche, publiés ou non, émanant des établissements d'enseignement et de recherche français ou étrangers, des laboratoires publics ou privés. 


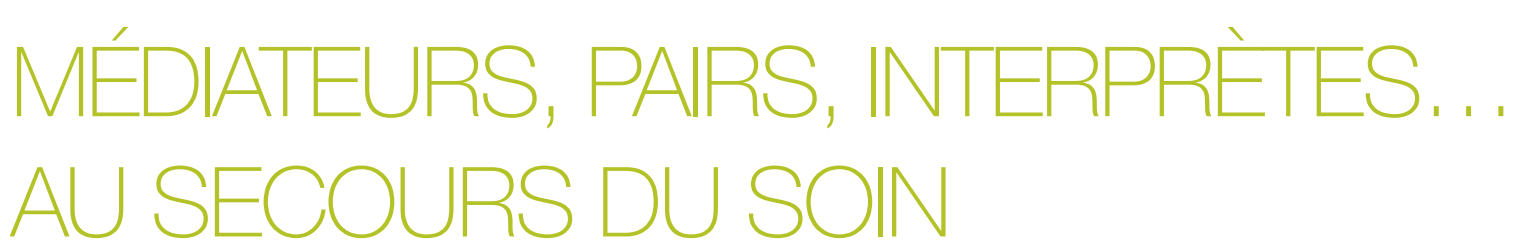

Une transformation plus ou moins tranquille traverse aujourd'hui les structures sanitaires et sociales. D'un côté, les métiers représentés au sein des établissements sanitaires, sociaux ou médico-sociaux se retrouvent "fragilisés». Des psychologues s'interrogent par exemple sur la pertinence de l'évolution de la psychiatrie publique - diffusion du concept de "rétablissement", développement de la réhabilitation psychosociale -, et sur leur place dans ces évolutions. Des soignants et des intervenants sociaux alertent également sur le manque de moyens pour mener à bien leur mission dans des structures en "crise ". D'un autre côté, se développent des métiers plus ou moins nouveaux (médiateur de santé-pair, interprète...) dont le nombre augmente chaque année. Ce développement est quantitatif, mais surtout qualitatif, puisque les formations qui sont proposées impliquent une "montée en compétences " de ces intervenants.

Si ces deux dynamiques ont pu apparaitre comme concurrentielles, elles tendent aujourd'hui à s'enrichir mutuellement. Les voix qui s'insurgeaient hier contre la présence des médiateurs de santé-pair dans les services de soins ou qui revendiquaient le fait de pouvoir travailler avec les personnes allophones sans interprète grâce à la mobilisation de référentiels théoriques sont de moins en moins audibles aujourd'hui. Dans les faits, elles sont surtout contredites par la réalité des pratiques dont les cadres normatifs se fragilisent, mais dont l'essence demeure intacte. La profusion des demandes d'intervention des "psys", notamment sur les terrains de la précarité, en témoigne. Par ailleurs, les métiers de la médiation participent du soin dans une perspective collaborative avec les personnes dont la profession pouvait revendiquer, par le passé, l'exclusivité de l'exercice de cette mission. En donnant voix à ce dernier argument, ce numéro de Rhizome documente les situations relevant des domaines de la santé mentale ou de l'intervention sociale, au sein desquelles des tiers de différents types interviennent. 
Une grande variété de cas de figure et de pratiques se déclinent au fil des articles, mais ces derniers révèlent surtout une diversité de points de vue, de regards et de perspectives. Ils participent à l'élaboration d'un prisme réflexif sur des questions qui apparaissent ainsi dans toute leur complexité. De la triangulation des instances dans le cadre des consultations avec interprète à la dissolution des catégories dans les situations de pair-aidance institutionnalisée, les différents articles nous invitent à lire des témoignages et des questionnements, notamment d'acteurschercheurs, sur les notions de "professionnalisation » et de " formation » - posées de l'extérieur et de l'intérieur -, des analyses et des réflexions, notamment sur l'expérience. Les perspectives sont plus ou moins objectivantes, subjectives, analytiques ou générales. Les observations faites se fondent un matériau expérientiel, introspectif ou déclaratif - dans le cas de travaux réalisés à partir d'entretiens -, sur des situations d'autoconfrontation ou sur des pratiques in situ. Soulignons deux éléments de contexte qui enracinent le développement de la médiation sanitaire, linguistique et sociale.

\section{De la pluralité des individus à la nécessité de la médiation}

Le processus contemporain d'individualisation, que ce soit dans une version positive - avec le mouvement continu d'émancipation -, ou négative - avec le processus de fragmentation sociale -, a accéléré l'hétérogénéisation de la société. II suffit de se rendre aux urgences d'un hôpital ou dans n'importe quelle administration pour s'en convaincre. Ce mouvement, qui ne peut que s'amplifier, touche aussi les domaines d'intervention auprès de populations précaires ou migrantes. Les catégories administratives (demandeur d'asile, titulaire du revenu de solidarité active [RSA]...) sont culturellement et socialement hétérogènes.

Le nombre de migrants primo-arrivants en France, par exemple, n'a cessé d'augmenter ces dix dernières années. Les demandes de soins des personnes migrantes sont nombreuses, notamment dans les permanences d'accès aux soins de santé (PASS) et auprès des équipes mobiles psychiatrie précarité (EMPP). Une controverse a émergé dans les institutions de soin quant à la prise en compte des spécificités du public migrant et à la nécessité de mettre en place - ou non - des dispositifs spécifiques ad hoc, comme l'ethnopsychiatrie dans le champ de la santé mentale. Si les tensions étaient fortes entre différents courants théoriques (rattachés à autant de structures) à la fin du xx $x^{e}$ siècle, elles tendent à s'estomper devant les attentes d'une prise en charge effective en santé et face à la multiplication d'initiatives pour proposer un soin adapté aux personnes migrantes, notamment grâce à la présence de médiateurs et/ou d'interprètes. Les acteurs de la puissance 
publique s'accordent aujourd'hui à dire que les personnes migrantes ne sont pas forcément un public spécifique, mais plutôt un public à besoins spécifiques. Ce recours aux tiers médiateurs paraît être une possibilité pour proposer un soin adapté et personnalisé sans mettre en place un soin spécialisé à telle ou telle catégorie administrative ou ethnique. D'ailleurs, la puissance publique promeut aujourd'hui le recours aux interprètes et médiateurs ${ }^{1}$ dans les équipes de soin et cherche à favoriser la professionnalisation et la spécialisation de ces acteurs.

De manière générale, l'individu paraît fragile tout en étant acteur de sa propre reconnaissance et est de moins en moins réductible à son statut institutionnel. Dans le paradigme d'action actuelle, que ce soit dans le champ sanitaire ou social, l'intervention se déploie dans une perspective de reconnaissance sociale des personnes en difficulté ou en souffrance. Pour ce faire, le recours à des instances, à des activités ou à des personnes à même de faire de la médiation est de plus en plus nécessaire. II ne s'agit donc plus seulement d'homogénéiser les profils individuels pour proposer un "traitement" sanitaire ou social. Dans un contexte de promotion de l'autonomie de l'usager ou du patient et de sa participation au processus de soin, et alors que tous les modes d'intervention sont de plus en plus personnalisés, ces médiations paraissent d'autant plus primordiales. L'injonction à devoir s'appuyer sur les forces, les compétences et les ressources des individus impacte les modalités de rencontre entre les accompagnants et les accompagnés, les soignants et les patients, qui peuvent nécessiter de recourir à des médiateurs (pairs) ou à des interprètes.

\section{L'efficience des pairs}

Si la " pair-aidance " se développe rapidement ces dernières années, c'est surtout qu'elle apparaît d'une grande aide aux personnes qui en bénéficient. II est difficile de se retrouver seul et le recours aux professionnels " non-pairs " n'est parfois pas

1 Selon l'article 90 de la loi $n^{\circ} 2016-41$ du 26 janvier 2016 de modernisation du système de santé français présente « la médiation sanitaire et l'interprétariat linguistique visent à améliorer l'accès aux droits, à la prévention et aux soins des personnes éloignées des systèmes de prévention et de soins, en prenant en compte leurs spécificités 》. suffisant. Le partage de ressources, de repères, d'idées, de questions et de savoirs est très utile et souvent efficace (Gardien, 2019). Ces savoirs ont été fréquemment appris ou construits sur la base de l'expérience de la vulnérabilité sociale et/ou de troubles de santé mentale. Ce partage d'expérience peut amener des "patients" ou des " usagers » à découvrir un savoir autre que celui du professionnel.

Différentes figures du pair peuvent se confondre ou coexister au sein des dispositifs, et elles interrogent le rapport au savoir ou à l'expérience en fonction des problèmes pratiques rencontrés - nécessité d'être compris ou traduit, besoin de rencontrer des personnes ayant les mêmes troubles. Si dans le domaine de l'intervention sociale des 
acteurs font la promotion des médiateurs-pairs, leur présence dans les dispositifs de soins destinés aux migrants pose d'autres problèmes, notamment eu égard à la reconnaissance - ou non - de l'inscription communautaire. En fonction de quelles compétences sont-ils choisis? Comment interviennent-ils dans la relation? De quoi sont-ils pairs? Le savoir ainsi constitué est-il transférable ou objectivable? Ces questions nous renvoient au débat autour de la formalisation et la formation des médiateurs, pairs et interprètes, thématisé à travers les articles à suivre.

\section{Médiateurs, pairs, interprètes}

Nous avions fait le choix, dans l'appel à contributions de ce numéro, de décloisonner les domaines de la pair-aidance telle qu'elle se rencontre dans le champ sanitaire, social et de l'interprétariat auprès des personnes migrantes, et ce pour deux raisons. La première est d'abord scientifique. Dans le cadre du projet de recherche "Réfugiés, migrants et leurs langues face aux services de santé " (Remilas), dont une partie du travail mené est restitué dans ce numéro en s'appuyant sur une perspective d'observation détaillée des pratiques in situ, nous avons effectivement constaté que la distinction historique et théorique entre " médiateur » et « interprète » est mise à l'épreuve des pratiques. En s'intéressant aux activités effectivement réalisées par les intervenants dans les consultations auxquelles ils prennent part, on remarque tout d'abord à quel point la notion de médiation - souvent réduite à l'idée d'apport d'informations culturelles qui pourraient apporter des éclairages ou à celle d'accompagnement dans la réalisation de démarches administratives - correspond en fait à une somme de multiples petites actions réalisées dans les situations (choix d'un mot, minute supplémentaire accordée après la séance, posture). Les articles montrent amplement que les interprètes s'apparentent de facto à des médiateurs. Le fait que ces champs d'activité dialoguent ensemble et que le lecteur puisse faire résonner les différentes contributions les unes avec les autres nous semble ainsi relever d'une dimension heuristique.

La seconde raison est plutôt d'ordre stratégique et politique. Fidèle à la ligne éditoriale de Rhizome, il importe moins de penser une psychiatrie, une psychologie ou une sociologie du "précaire ", du " migrant " ou du "malade ", que de questionner en quoi la prise en compte de ces publics recalibre les modalités d'intervention sociale ou en santé. II s'agirait donc d'étudier ces dernières en les envisageant comme un laboratoire de pratiques qui sont amenées à se développer à mesure de l'évolution de la société, de son individualisation et de son anormalisation. En effet, nous faisons de plus en plus le constat que le savoir purement technique est insuffisant. À partir de là, les médiations humaines sont appelées à se développer, en particulier pour les personnes en situation de précarité et/ou de migration. 


\section{Pair-aidance et rétablissement}

Ce numéro de Rhizome commence et se termine par deux articles avec une écriture singulière, où les auteurs rendent compte de leurs expériences. En écho à l'individualisation évoqué précédemment, le numéro reflète deux positions : l'une cherche à trouver des récurrences et des similarités, soit des éléments pouvant être généralisés à l'ensemble des pratiques, et l'autre mettant l'accent sur l'unique, le cas, ce qui échappe au commun. Cela pourrait aussi être perçu comme une tension entre le singulier et le général.

Camille Niard, Philippe Maugiron et Nicolas Franck lient le développement de la pair-aidance à celui du concept et des pratiques de rétablissement. Ce qui est défendu par les médiateurs, c'est le fait qu'ils amènent l'espoir, qu'ils démontrent que le "rétablissement est possible » et qu'il est possible "d'avoir un projet de vie malgré la maladie ». La perspective portée par les médiateurs recouvre, à notre sens, celle de nombreux cliniciens. Camille Niard évoque une " écoute profonde, sensorielle et une attention particulière à la communication non verbale ". Elle se définit aussi comme "passeuse ». Elle affirme notamment : "On ne se décrète pas pair de quelqu'un. On le découvre lors de la rencontre. [...] Par sa posture de tiers, à la fois professionnel au sein d'une équipe et concerné, le médiateur pair encourage à dépasser les étiquettes, à sortir du cadre établi, de manière sécurisée. " Ces témoignages font d'ailleurs écho de ce que les intervenants en santé mentale disent généralement de leurs pratiques.

Les articles d'Aurélien Troisœufs et de Lise Demailly impriment la valorisation des savoirs expérientiels. Un véritable changement de paradigme traverse ainsi les structures sanitaires. L'expérience de la souffrance, de la maladie, de l'hospitalisation, du rétablissement et des "petits trucs " peuvent être partagés sans qu'ils ne correspondent forcément à un savoir académique ou scientifique. Émilie Charlier présente un groupe de parole en Belgique avec des " experts du vécu ", facilitateurs " garants du cadre ". Les uns peuvent s'identifier aux histoires des autres. Encore une fois, ce type d'analyse peut faire écho à ce que certains psychologues disent de leurs activités, notamment en mobilisant des théories analytiques et groupales.

\section{Statuts en tension}

Laëtitia Schweitzer nous propose un écrit, dissonant ou, du moins, empreint d'une forte réflexivité. Elle affirme qu'«il ne faut pas céder à une vision positiviste du travail pair qui produirait des effets en soi, dans un absolu débarrassé de toute 
contingence et de toute caractéristique situationnelle ». Le développement du travail pair s'inscrit d'ailleurs dans une forme de précarisation des champs du travail social et sanitaire. Elle note aussi qu'il existe une " confusion entre travail pair et la participation des personnes accompagnées : les travailleurs pairs seraient implicitement les représentants de celles-ci, dont ils porteraient la voix dans les instances d'organisation de l'accompagnement médico-social, au nom de leur parité avec elles ". De manière générale, les articles sur les médiateurs-pairs ne portent pas tant sur les pratiques que sur les questions liées à leurs statuts.

Quant à la série d'articles qui portent sur les situations avec interprètes, ils relèvent de témoignages sur leur pratique propre, de réflexions sur la base d'entretiens ou d'analyses de consultations. Les grandes questions qui traversent ces contributions, notamment celles de la neutralité de l'interprète, sa place dans l'interaction en tant que participant, la confiance que les autres protagonistes lui font ou non, reçoivent des réponses étonnamment convergentes, auxquelles le prisme des points de vue et des éclairages donne de l'épaisseur. Émane ainsi de tous les articles le constat que l'interprète effectue bien un travail de médiation et qu'il ne se conforme pas à la norme de neutralité pourtant enseignée et répertoriée comme bonne pratique. Toutefois, les articles montrent également que la question n'est pas si binaire : l'interprète étant un participant engagé dans une situation, il adapte sa pratique à ce qui se passe, comme le font aussi ses interlocuteurs. Cela explique donc les changements, les fluctuations, les oscillations et les balancements repérés par les auteurs.

\section{L'interprète neutre et transparent?}

L'article d'Elizaveta Chernyshova et d'Anna Claudia Ticca porte sur une consultation de psychiatrie en présence d'un interprète et questionne l'image de "l'interprète machine " qui est souvent avancée comme le modèle idéal de la neutralité. Sur la base d'une analyse détaillée de deux extraits, l'article met en évidence comment l'analyse du déroulement " pas à pas " de l'interaction montre que l'interprète oscille entre deux postures, celle de la translation machine (Bot, 2005) et celle du participant à part entière, s'affirmant comme interlocuteur ayant une voix et une présence dans l'interaction, voire comme un cothérapeute. L'article montre aussi très clairement que la qualité de l'interprétation n'est pas la responsabilité exclusive du tiers, mais qu'elle est le résultat d'une entreprise collective.

La même question de la neutralité, ici pourrait-on dire la transparence de l'intervention de l'interprète, se retrouve dans l'article de Vanessa Piccoli et de Véronique Traverso. Leur contribution s'inscrit dans l'idée qu'au-delà des particularités, des 
spécificités et des cas, il est possible d'identifier des pratiques discursives récurrentes dans les consultations avec interprètes, dont la description détaillée constitue un apport pour la formation, la réflexion sur les bonnes pratiques et les standards. À cette fin, l'article décrit un procédé observé dans les consultations de psychologie avec des personnes migrantes, qui consiste pour l'interprète, dans sa traduction de la description que le patient fait de son état affectif (passé ou présent), à commenter les choix lexicaux du patient et la non-équivalence entre les langues. Par ce procédé, l'interprète déroge à la fameuse neutralité et égratigne l'illusion de la transparence du langage.

C'est encore cette question que l'on entend dans la contribution d'Anne-Marie Cervera, qui, sur une base introspective, reconstitue les processus de réflexion qu'en tant qu'interprète elle est appelée à mettre en œuvre dans sa pratique professionnelle. Elle montre, dans la reconstitution de ce parcours intérieur, à la fois les difficultés liées à l'établissement de la confiance du patient ainsi que la complexité de la traduction en santé mentale qui se dissimule sous une apparente simplicité du langage. De plus, et surtout, l'article dévoile l'enchaînement de décisions qu'elle est amenée à prendre à chaque instant de l'interaction, non seulement pour choisir le sens et traduire, mais aussi pour revenir, par différents procédés, sur une traduction déjà produite lorsque la suite de l'interaction montre qu'elle ne correspondait pas à ce que le patient voulait dire initialement.

\section{L'interprète : un participant à part entière}

Si la question de l'interprète comme participant à part entière était déjà présentée à travers la mise en cause de la neutralité, celle-ci se retrouve, dans une seconde série d'articles, au centre des réflexions à travers les questions de la confiance, des modalités de collaboration et de l'adaptation continue des modes d'intervention de l'interprète aux contingences de la consultation.

Iona Atger, Djamel Khouas et Stéphanie Larchanché rapportent une recherche-action dont l'objectif était d'améliorer la collaboration entre professionnels de santé mentale et interprètes professionnels. L'article discute, à partir de cette expérience, un grand nombre des difficultés mentionnées par les professionnels pour travailler avec des interprètes, soit les questions de confiance, de perte de contrôle ou de temps, ainsi qu'une fois encore, de la neutralité. Sur ce dernier point, à l'unisson des précédents articles, les auteurs considèrent que, si celle-ci demeure toujours une quête pour l'interprète, " elle devient chimère " dans le champ de la santé mentale et ils avancent l'idée selon laquelle la position de l'interprète devient optimale quand elle 
est mouvante. Leur expérience les conduit à postuler que le passage d'une posture de méfiance à une posture de confiance nécessite une formation ou un accompagnement simultané de l'interprète et du professionnel. Ils ouvrent enfin une perspective nouvelle sur la question du temps de consultation, censé être allongé par la présence de l'interprète, en y voyant un moment pour réfléchir, pour s'observer, pour deviner le sens des propos dans la langue de l'autre.

Orest Weber et Florence Faucherre présentent le dossier pédagogique en ligne qu'ils ont élaboré à partir d'analyses - combinant approches cliniques et discursives - d'enregistrements vidéo de consultations d'investigation psychiatriques avec interprètes. L'article identifie six défis pour les interprètes. Ces difficultés apparaissent de façon récurrente (telles que les mentions d'émotion, les productions peu audibles), puis les auteurs montrent comment les défis sont abordés dans le dossier pédagogique, sur la base d'extraits de consultation qui les illustrent, de leur analyse et de la formulation de conseils et de stratégies pouvant être efficaces dans ces cas.

En résonance avec les articles d'Elizaveta Chernyshova et d'Anna Claudia Ticca ainsi qu'avec celui d'Anne-Marie Cervera, Anne-Sophie Haeringer problématise la question des choix de traduction de l'interprète, cette fois sous l'angle des risques qui leur sont inhérents. Elle observe, en effet, comment une solution trouvée pour pallier une difficulté rencontrée à un certain moment est susceptible de créer un problème par la suite.

Amandine Bachini, Élodie Berenguer, Hachimia Abdallah, Sitti Demassi, Zaliffa Gue et Moinamaoulida Kassim rapportent la situation dans un centre médicopsychologique (CMP) à Mayotte où la traduction est assurée par des agents de service hospitalier (ASH) mahoraises, qui ne bénéficient d'aucune formation spécifique ni de reconnaissance institutionnelle pour leurs compétences d'interprète. L'article montre bien que la triangulation de la relation thérapeutique opérée par la présence des interprètes amène des moments de pause propices à la réflexion, crée une autre temporalité avec des temps de respiration et d'échanges qui fédère une secondarisation des processus psychiques. Parallèlement, il soulève la question de la place et du statut des ASH qui interprètent, ainsi que de l'invisibilisation de leur rôle dans le soin, tout en mettant en évidence la hiérarchie existant entre les savoirs (savoirs académiques cliniques des professionnels de soin francophones et savoirs expérientiels et culturels, véhiculés par les langues locales). Ce questionnement est mis en parallèle avec celui de l'héritage colonial, les auteurs avançant le terme d'ambivalences " cicatricielles " quant à la reconnaissance du statut socioprofessionnel des $\mathrm{ASH}$-traductrices mahoraises dans le service. 
L'article d'Ada Luz Duque, basé sur son expérience d'interprète professionnelle, part quant à lui du constat que faire appel à un interprète est généralement le résultat d'une nécessité de compréhension linguistique plutôt que d'une réelle voIonté de constituer un accompagnement pluridisciplinaire. À partir de là, elle questionne la place de l'interprète dans la triade soignant-patient-interprète, insistant sur celle de l'interprète comme participant, voire cothérapeute, qui apporte aussi - et pourrait-on dire inévitablement - son interprétation et ses affects. L'auteure montre également que le caractère fluctuant et nécessairement improvisé de la pratique de l'interprète est toujours adapté à ce qui se passe, alors qu'il est parfois analysé de manière trop figée. Elle regrette ainsi que la possibilité de construire ensemble les pratiques et le soin dans la triade, et de manière non standardisée, soit rarement considérée.

Nicolas Chambon et Roman Pétrouchine s'intéressent eux, à travers une analyse de cas, à la manière de rendre compte, d'objectiver et de traduire un "événement traumatique dans une situation d'expertise médicale en présence d'une interprète professionnelle ". L'activité de médiation est envisagée dans des enjeux globaux, où les savoirs que partagent la patiente et ses tiers (interprète et intervenante sociale), concernent d'autres logiques que celle du médecin, qui se doit cependant d'en tenir compte.

\section{Expériences et concernement}

L'article de Gwen Le Goff et de Natacha Carbonel reprend différentes questions récurrentes soulevées par les situations avec les interprètes en les abordant du point de vue des personnes migrantes. Elles mettent en évidence aussi bien leurs attentes vis-à-vis des interprètes, y compris en termes affectifs ou relationnels, et les stratégies de résistance (rejet de la dépendance induite, absence de confiance) mises en œuvre pour s'affranchir de la nécessité de recourir à eux. II est frappant, et sans doute serait-il intéressant de faire savoir et comprendre aux professionnels, ce reflet inversé de leurs propres inquiétudes et difficultés. L'article thématise également ce désir de vouloir partager l'expérience du vécu à d'autres, notamment en devenant interprète.

On retrouve cette même volonté dans l'article d'Olivia Gross qui questionne les motivations des candidats à la fonction de "médiateur santé-pair ", particulièrement parce qu'elle leur permettrait " de mobiliser diverses compétences et de trouver du sens à leur parcours de vie »". L'auteure invite à la réflexivité sur le cadre de l'exercice des médiateurs. Ce questionnement peut rejoindre celui de nombreux 
professionnels qui s'interrogent sur l'exercice et les frontières de leur métier, et plus précisément sur le sens de l'intervention. Cette réflexion, abordée par Élodie Gilliot et Mathilde Sorba, est au cœur de l'évolution des pratiques dans l'orientation prise par des structures dans le cadre de la politique dite de "logement d'abord". Elles interrogent " les conséquences pratiques d'une nouvelle répartition des rôles entre l'institution, les intervenants sociaux et les personnes accompagnées ". Elles défendent ainsi l'idée que " l'activité de médiation qui en résulte, et se déploie de façon très pragmatique, n'est pas réduite à un rôle de pacification des relations entre institutions et usagers, mais elle se présente plutôt comme une activité qui consiste à permettre une rencontre, ou à adapter des réponses institutionnelles d'ordre techniques, juridiques, matérielles, un environnement social à une personne qui désire, espère... »

Christian Laval et Ève Gardien défendent quant à eux l'importance de l'entraide et de l'échange de savoirs entre pairs et le risque d'une dilution de ce savoir " pair " au profit d'une fonction de médiation, qui se prête mieux à être reconnue par la puissance publique. Ce numéro se conclut d'ailleurs par une présentation, par Graziella Golf, Thomas d'Hauteville et Magali Molinié, d'un groupe du " Réseau sur l'entente de voix " (REV) qui thématise les apports de l'entraide pour des personnes qui partagent des expériences sensibles parfois communes, et problématise alors la fonction de facilitateur de ces groupes. Ils affirment que " la rencontre entre pairs permet de sortir de son isolement, de se réapproprier son expérience, de lui chercher un sens".

Nous vous invitons alors à lire ou relire ce Rhizome comme un espace de rencontre qui offre la possibilité de nous (re)questionner collectivement sur ce que sont le soin et ses pratiques. D

\section{BIBLIOGRAPHIE}

Bot, H. (2005). Dialogue interpreting in mental health. Amsterdam/New York : Rodopi (Utrecht Studies in Language and Communication, 19).

Gardien, E. (dir.) (2019). L'accompagnement par les pairs. Une approche transversale aux champs de la santé, de la santé mentale et du handicap. Actes de la Journée d'étude Expairs. Rennes, 7 novembre 2018 . 